\title{
Discovering Undisclosed Paid Partnership on Social Media via Aspect-Attentive Sponsored Post Learning
}

\author{
Seungbae Kim, Jyun-Yu Jiang and Wei Wang \\ Department of Computer Science, University of California, Los Angeles \\ Los Angeles, CA, USA \\ \{sbkim,jyunyu,weiwang\}@cs.ucla.edu
}

\begin{abstract}
The transparency issue of sponsorship disclosure in advertising posts has become a significant problem in influencer marketing. Although influencers are urged to comply with the regulations governing sponsorship disclosure, a considerable number of influencers fail to disclose sponsorship properly in paid advertisements. In this paper, we propose a learning-to-rank based model, Sponsored Post Detector $(S P O D)$, to detect undisclosed sponsorship of social media posts by learning various aspects of the posts such as text, image, and the social relationship among influencers and brands. More precisely, we exploit image objects and contextualized information to obtain the representations of the posts and also utilize Graph Convolutional Networks (GCNs) on a network which consists of influencers, brands, and posts with embed social media attributes. We further optimize the model by conducting manifold regularization based on temporal information and mentioned brands in posts. The extensive studies and experiments are conducted on sampled real-world Instagram datasets containing 1,601,074 posts, which mention 26,910 brands, published over 6 years by 38,113 influencers. Our experimental results demonstrate that SPoD significantly outperforms the existing baseline methods in discovering sponsored posts on social media.
\end{abstract}

\section{CCS CONCEPTS}

-Information systems $\rightarrow$ Data mining; Social networks; • Applied computing $\rightarrow$ Marketing.

\section{KEYWORDS}

Influencer marketing; Sponsorship detection; Aspect attention; Graph convolutional networks; Multimodal learning

ACM Reference Format:

Seungbae Kim, Jyun-Yu Jiang and Wei Wang. 2021. Discovering Undisclosed Paid Partnership on Social Media via Aspect-Attentive Sponsored Post Learning. In Proceedings of the Fourteenth ACM International Conference on Web Search and Data Mining (WSDM '21), March 8-12, 2021, Virtual Event, Israel. ACM, New York, NY, USA, 9 pages. https://doi.org/10.1145/3437963. 3441803

Permission to make digital or hard copies of all or part of this work for personal or classroom use is granted without fee provided that copies are not made or distributed for profit or commercial advantage and that copies bear this notice and the full citation on the first page. Copyrights for components of this work owned by others than the author(s) must be honored. Abstracting with credit is permitted. To copy otherwise, or republish, to post on servers or to redistribute to lists, requires prior specific permission and/or a fee. Request permissions from permissions@acm.org.

WSDM '21, March 8-12, 2021, Virtual Event, Israel

(c) 2021 Copyright held by the owner/author(s). Publication rights licensed to ACM. ACM ISBN 978-1-4503-8297-7/21/03 . \$ \$15.00

https://doi.org/10.1145/3437963.3441803

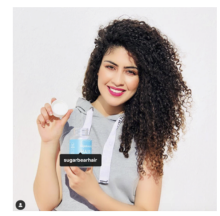

@sugarbearhair has keeping my hair popping for 6 months now, just take theses cute vitamins and let your hair do the talking. \#sugarbearhair

Figure 1: An example of paid media that fails to disclose sponsorship. Despite the influencer advertises a product and mentions a certain brand name, no sponsorship is disclosed.

\section{INTRODUCTION}

Influencer marketing has been gaining significant attention from marketers as an essential advertising method recently [23]. As the rapid growth of the influencer marketing industry results in numerous paid advertisements in social media, the transparency issue of advertising posts has been raised. According to the regulations from the Federal Trade Commission (FTC) [9], the Advertising Standards Authority (ASA) [3], and the Organisation for Economic Co-operation and Development (OECD) [13], influencers are required to conspicuously disclose sponsorship when they publish paid advertisements. That is, mentioning brand names and the relationship between a mentioned brand and an influencer in paid advertisements, thereby having transparency in advertising posts. However, a noticeable number of influencers fail to disclose paid partnerships with brands in their advertising posts, either because they are not aware of the regulations [2] or because they are concerned about lowering the effectiveness of the advertisement [12]. Surprisingly, the recent survey [2] reveals that only $52 \%$ of influencers and $60 \%$ of marketers have a good understanding of the regulation. This implies that the lack of legal knowledge and education for social media users can lead to social issues amid the rapid growth of social media. Figure 1 shows an example of a paid media where the influencer advertises the product of the brand in the absence of mentioning sponsorship.

The sponsored posts without disclosing the sponsorship may cause the following problem. Audiences will be increasingly skeptical toward the influencers' posts, and hence influencers lose the trust. Influencer marketing can only become effective when people think that influencers are trusted sources of information [21]. Furthermore, the lack of transparency in the advertising posts can negatively impact brand image [12]. For the steady growth of the influencer marketing industry with proper advertising practice, the FTC has monitored and warned a few famous celebrities in social media who violated the endorsement regulations ${ }^{1}$. However, it is impractical to monitor the millions of influencers on social media.

\footnotetext{
${ }^{1}$ https://www.ftc.gov/news-events/press-releases/2017/04/ftc-staff-remindsinfluencers-brands-clearly-disclose
} 
Although influencer marketing has gained noticeable attention recently, only a limited number of studies focused on sponsorship disclosure in influencer marketing. Some previous works examine the effect of the presence of sponsorship in social media [12, 31, 37] and suggest that disclosing sponsorship helps audiences to identify the post as an advertisement but lowers purchase intention. Moreover, Wojdynski et al. [35] attempt to measure the sponsorship transparency of paid advertisement by considering audiences' perceptions. While the previous works discuss the importance of detecting undisclosed sponsorship of social media posts, no study has proposed a method yet. Additionally, the previous works solely rely on a small number of survey results, thereby lacking evaluation with a large dataset.

In this paper, we propose a learning-to-rank based model, Sponsored Post Detector ( $S P o D$ ), that can detect sponsorship of social media posts. Our model incorporates three different aspects (i.e., modalities) on social media including graph, text, and image to represent the social media posts. We first employ the Graph Convolutional Networks (GCNs) [20] to leverage the characteristics of posts and the social relationship among influencers and brands. To adopt GCNs, we construct a heterogeneous network that connects influencers, posts, and brands. Besides the graph features, we also generate image and text features of each post to further describe the characteristics of the posts. Particularly, we use the pre-trained Inception-V3 model to obtain the image object features which have 1,000 categories [32] and utilize BERT [10] to create contextualized features of social media post captions. Moreover, we apply attention [34] over the three sets of features to estimate the importance of each aspect of social media posts, thereby utilizing more important aspects to detect hidden sponsorship. In addition to the attentive post features, we conduct a manifold regularization method to optimize the model performance. More specifically, we propose to exploit posting time and mentioned brands from social media posts for temporal regularization. For example, we place more weight on posts created at similar times and mentioning the same brand, that is, posts that likely belong to the same marketing campaign. With the proposed temporal regularization, our model takes the attentive post features as input to rank given social media posts by their sponsorship scores.

We summarize our contributions as follows:

- To the best of our knowledge, this is the first attempt to rank social media posts by their sponsorship scores. We believe that our proposed SPoD can be beneficial for marketers and government organizations to find brands and influencers in violation of endorsement guides $[3,9,13]$, thereby protecting consumers. Besides, social media platforms can utilize SPoD to help users recognize the sponsorship disclosing regulation. As SPoD exploits the prevalent social media features, our model can be practically adopted to any social media.

- We propose a novel learning-to-rank model, SPoD, that incorporates with texts, images, and relationships among influencers and brands. Moreover, we estimate the importance of the different modalities by using attention to acquire decent post representation. Besides, we employ the redundancy between the posts and their published times to learn temporal sponsored relationships.
We conduct extensive experiments on a real-world dataset collected from Instagram which is known as the most popular social media for influencer marketing [23]. Our extensive experimental results demonstrate that $\mathrm{SPoD}$ improves performance by $54.3 \%$ in detecting undisclosed sponsorship compared to the best baseline method.

- Our analysis further reveals that the text features significantly improve the ranking performance. We find that contextualized features improve SPoD by $53.8 \%$. We also observe that SPoD properly ranks the posts which are in various caption lengths. Particularly, SPoD obtains $216 \%$ improvement over the state-ofthe-art text baseline method with very short caption posts by taking advantage of the graphical structural information.

- To make our research reproducible and benefit the whole research field, we release the proposed model and the benchmark dataset ${ }^{2}$. Specifically, the dataset consists of 38,113 influencers and their $1,601,074$ posts which mention 26,910 brand names.

\section{PROBLEM STATEMENT}

In this section, we formally define the goal of this paper. Suppose we have a set of posts $P=\left\{p_{n}\right\}_{n=1}^{|P|}$ published by a set of users $U=\left\{u_{m}\right\}_{m=1}^{|U|}$. Each post can be represented as $p_{n}=\left(t_{n}, a_{n}, b_{n}, l_{n}\right)$ where $t_{n}, a_{n}, b_{n}$, and $l_{n}$ denote text, image(s), mentioned brand(s), and posting time of the post, respectively. Note that a post mentions at least one brand where the brand is in the set of brands $B=$ $\left\{b_{k}\right\}_{k=1}^{|B|}$. Given text and images of a post, we extract text features and image features, $X^{T} \in \mathbb{R}^{n \times f}$ and $X^{I} \in \mathbb{R}^{m \times g}$, respectively, where $f$ and $g$ are the numbers of features. Moreover, we have graph features, $X^{G} \in \mathbb{R}^{n \times h}$, where $h$ is the number of features. The graph features can be generated from a heterogeneous network $\mathcal{G}=(E, V)$ where the vertices $V=(U, P, B)$ are composed of users, posts, and brands, respectively. Given a set of posts $P$, we aim to rank the posts by learning the distinctive features of sponsored posts so that sponsored posts with the absence of sponsorship disclosure can be discovered.

\section{METHODOLOGY}

\subsection{Framework Overview}

Here we briefly give an overview of our proposed framework, SPoD, as shown in Figure 2. To leverage multimodal inputs of social media posts, we utilize three encoders, including graph encoder, text encoder, and image encoder. The graph encoder takes the heterogeneous network that includes users, posts, and brands as an input. Moreover, each node in the graph has a set of features as contextual representations to indicate the entity characteristics. Based on the heterogeneous structures of different entities and their features, graph convolutional networks (GCNs) are applied to derive appropriate node representations. In addition to GCN-encoded features, the text and image of each post are encoded by a contextualized text encoder and an image encoder, respectively. We then apply attention [34] over the three sets of features to estimate their importance and generate the post representations. The sponsorship scores of candidate posts are then computed based on all of the corresponding features and ranked for discovering sponsored posts.

${ }^{2}$ https://github.com/ksb2043/WSDM21_Sponsored-Post-Detector-SPoD 


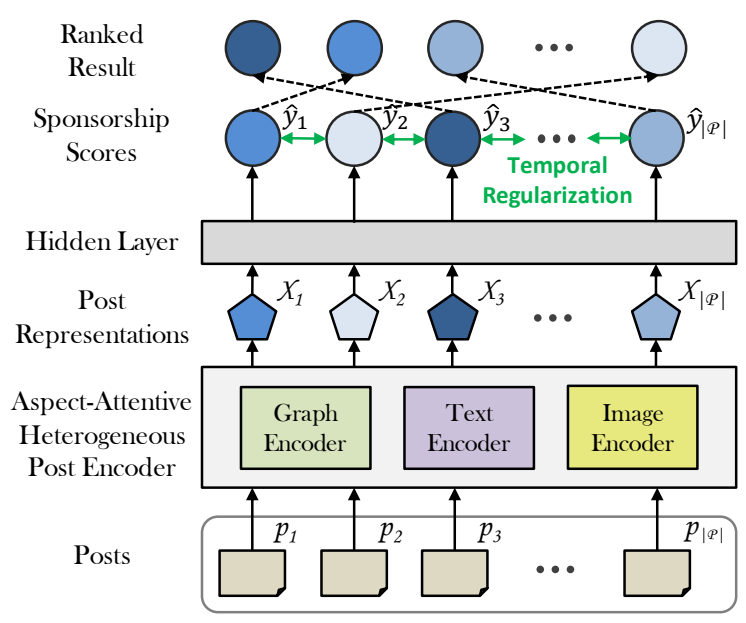

Figure 2: The overall framework of the SPoD. The aspectattentive heterogeneous post encoder generates post representations. The estimated sponsorship scores are optimized by conducting temporal regularization for detecting sponsored posts.

Finally, we optimize the sponsorship scores by conducting temporal regularization based on posting time and mentioned brands from the input post set.

\subsection{Aspect-Attentive Heterogeneous Post Encoder}

To acquire decent representations of posts, we utilize three encoders including the graph, text, and image encoders to capture knowledge from different aspects, and apply aspect-attention over the three sets of features as shown in Figure 3.

3.2.1 Graph Encoder. To model posts with the graphical structure, we first construct a heterogeneous network and then apply graph convolutional networks (GCNs) [20] to derive GCN-encoded features for each candidate post.

Heterogeneous Network Construction. To construct a heterogeneous network, we consider three different entities including posts, users (i.e., influencers), and brands mentioned in posts. Note that the constructed heterogeneous network in our framework can be flexibly expanded with any additional relevant entities. The edges in the heterogeneous network indicate the interactions between entities behind nodes. The node of each post is linked to the node of the author user. If a brand is mentioned in a post, the post node has an edge to the brand node. Note that since more than one brand can be mentioned in a post, a post node can have multiple edges to brand nodes. More specifically, the edges of the network are represented by a sparse matrix $A \in \mathbb{R}^{N \times N}$, where $A_{i j}=1$ if the $i$-th and $j$-th nodes are connected; otherwise, $A_{i j}=0$.

Node Features. Each node in the network has a set of features while all of the features can be represented as

$$
Z=\left[Z^{P} ; Z^{U} ; Z^{B}\right] \in \mathbb{R}^{N \times d},
$$

where $Z^{P}, Z^{U}$, and $Z^{B}$ are the features of nodes for posts, influencers, and mentioned brands, respectively; $N$ and $d$ are the number

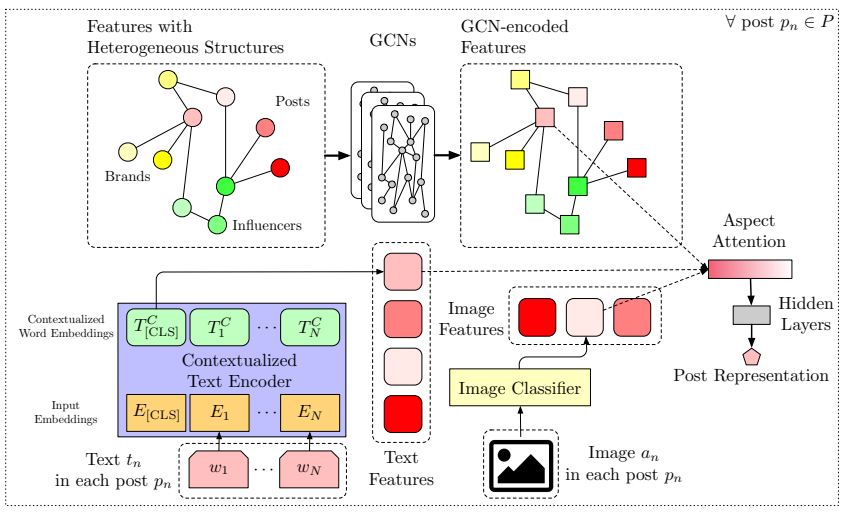

Figure 3: The illustration of the post encoder using heterogeneous information.

of all nodes in the network and the number of features for each node, respectively. The detailed features in this paper are defined in Section 3.2.5.

Graph Convolutional Networks To leverage the knowledge of structural information, we propose to apply GCNs to encode node representations with both node features and network structures. First, the adjacency matrix $\boldsymbol{A}$ is transformed into a normalized adjacency matrix $\hat{A}$ as follows:

$$
\hat{A}=D^{-\frac{1}{2}} A D^{-\frac{1}{2}},
$$

where $D$ is the diagonal matrix of node degrees. GCNs can then be operated based on the normalized adjacency matrix $\hat{A}$ and the feature matrix $Z$.

To model complicated network structures, we consider multilayer GCNs by propagating information through different layers. Formally, the outputs of the $i$-th layer in GCNs, $H^{(i)} \in \mathbb{R}^{N \times k}$, can be computed as follows:

$$
H^{(i)}=\sigma\left(\hat{A} H^{(i-1)} W^{(i-1)}\right),
$$

where $k$ is the number of hidden dimensions in GCNs; $\boldsymbol{H}^{(\boldsymbol{i}-1)}$ is the outputs of the previous layer; $W^{(i-1)}$ is a matrix of layerspecific trainable weights; $\sigma(\cdot)$ is a nonlinear activation function. Note that $\boldsymbol{H}^{(0)}=Z$ as the base case. Finally, the GCN-encoded representations $X$ can be computed by concatenating the outputs of different layers as follows: $X^{G}=\left[H^{(1)}, H^{(2)}, \ldots, H^{(L)}\right]$, where $L$ is the number of layers in GCNs.

3.2.2 Text Encoder. To model contextualized knowledge from text in posts, we encode the text $t_{n}$ in a given post $p_{n}$. Instead of learning from scratch, we apply the pre-trained state-of-the-art neural language model, BERT [10]. Note that any potential language model can be applied to the text encoder. Given the text in a post $t_{n}$, a length $j$ sequence of words $c=\left[c_{1}, c_{2}, \cdots, c_{j}\right]$, BERT adds an initial token [CLS] to alleviate the positional bias in input embedding $c^{\prime}=\left[[\mathrm{CLS}], c_{1}^{\prime}, c_{2}^{\prime}, \cdots, c_{j^{\prime}}^{\prime}\right]$. The input word sequences of $n$ posts, $\mathbb{Q}^{\left(j^{\prime}+1\right) \times n}$, then goes to the transformer $\mathbf{F}_{T}$ with $o$ layers. We generate the output of the $o$-th transformer $\mathbf{F}_{T}\left(\mathbf{F}_{T}^{o-1}\right)=D^{o}=\left[d_{[C L S]}^{o}, d_{1}^{o}, d_{2}^{o}, \cdots, d_{j^{\prime}}^{o}\right]$ as the representation 
of each word in the word sequences. Finally, we only adopt the $d_{[C L S]}^{o}$ to form the text representation $X^{T}$.

3.2.3 Image Encoder. In addition to the graph and text, we use images attached in the posts since images are known as one of the most important factors in effectively advertising products in social media marketing [24]. For example, the influencer in Figure 1 holds the product in the image for advertising purposes. We apply the pre-trained Inception-V3 model trained with onemillion images in 1,000 object categories [32] to avoid training images from scratch. Particularly, the image encoder takes input images from a post and generates a feature vector with $g$ dimensions where each dimension represents the probability that the image contains the corresponding object. That is a list of $s_{n}$ images from a post $p_{n}, a_{n}=\left[a_{n 1}, a_{n 2}, \cdots, a_{n s}\right]$. We use the maximum value for each dimension while aggregating the feature vectors as follows: $\mathbf{F}_{I}\left(a_{n}\right)=\max -\operatorname{pool}\left(\left\{a_{n i} \mid 1 \leq i \leq s_{n}\right\}\right)$, where the function max-pool $(\cdot)$ remains the maximum value for each dimension over the feature vectors of $s_{n}$ images. Finally, the image representation $X^{I}$ contains the image object vectors from $n$ posts.

3.2.4 Aspect-Attention. To estimate the importance of features from different aspects, including the heterogeneous graph, texts, and images, we apply the attention mechanism over the three sets of features. We first apply a fully-connected hidden layer to each set of features to have the same dimension of the features as:

$$
V^{G}=\mathcal{F}_{G}\left(X^{G}\right), V^{T}=\mathcal{F}_{T}\left(X^{T}\right), V^{I}=\mathcal{F}_{I}\left(X^{I}\right),
$$

where $\mathcal{F}_{G}(\cdot), \mathcal{F}_{T}(\cdot)$, and $\mathcal{F}_{I}(\cdot)$ are fully-connected layers for the graph, text, and image features, respectively. The sets of features from different modalities can be represented as:

$$
V=\left[V^{G}, V^{T}, V^{I}\right]
$$

The importance $\alpha_{i}$ of feature $V_{\boldsymbol{i}}$ can be estimated as:

$$
\alpha_{i}=\frac{\exp \left(\boldsymbol{r}_{\boldsymbol{i}} \cdot \boldsymbol{r}^{\boldsymbol{c}}\right)}{\sum_{j} \exp \left(\boldsymbol{r}_{j} \cdot \boldsymbol{r}^{\boldsymbol{c}}\right)},
$$

where $\boldsymbol{r}_{\boldsymbol{i}}=\tanh \left(\mathcal{F}\left(V_{i}\right)\right)$ is the hidden representation of the feature $V_{i} ; \mathcal{F}(\cdot)$ is a fully-connected layer; $\tanh (\cdot)$ is the activation function; $\boldsymbol{r}^{c}$ is the context vector for importance estimation. The estimated score $\alpha_{i}$ is multiplied with corresponding features $\boldsymbol{V}_{\boldsymbol{i}}$ to get weighted values, and the representation of the post can be derived by taking all the weighted values of the graph, text, and image features as follows:

$$
X=\sum_{i} \alpha_{i} \cdot V_{i}
$$

3.2.5 Node Features in GCNs. To represent characteristics of nodes in the network, we incorporate four types of node features, including node type, influencer, post, and brand as shown in Table 1. Note that our proposed framework is not limited to these features, therefore, any potential feature can be additionally applied to the model.

- Node type features. To indicate one of the three types of nodes, including post, influencer, and brand, we apply the one-hot coded node type feature in this category.
Table 1: Node features that represent characteristics of each type of node in GCNs.

\begin{tabular}{|c|c||l|}
\hline Category & Feature & Description \\
\hline \hline Node & Node Type & Node type in the heterogeneous network. \\
\hline \multirow{4}{*}{ Influencer } & Keywords & The normalized frequency of keywords. \\
& Followers & Number of followers. \\
& Followees & Number of followees. \\
& Posts & Number of published posts. \\
& Influncer Category & Major interest of the influencer. \\
\hline \multirow{5}{*}{ Posts } & Likes & Number of likes in a post. \\
& Comments & Number of comments in a post. \\
& Hashtags & Number of hashtags(\#) in a post. \\
& Usertags & Number of usertags(@) in a post. \\
& Caption Length & Length of text in a post. \\
& Images & Number of images in a post. \\
& Posting Day & The day a post was published. \\
\hline \multirow{5}{*}{ Brand } & Followers & Number of followers. \\
& Followees & Number of followees. \\
& Posts & Number of published posts. \\
& Brand Category & Business type of the brand. \\
\hline
\end{tabular}

- Influencer features. We exploit the normalized frequency of keywords to capture textual patterns of influencers. We select the frequently used keywords based on their Chi-square values. Note that we use the top 100 keywords in this study. We also use the number of followers, followees, and published posts features that represent the reputation of influencers [18]. Moreover, we exploit the major interest of the influencers such as Food and Interior [19].

- Post features. To represent post characteristics, we exploit the features which are widely used in any social media. We first obtain the numbers of likes and comments in a post which can represent the popularity of the given post. Since it is well known that people tend to avoid advertising $[8,17]$, such post popularity features can help provide a distinguishable representation of sponsored posts. We also employ the numbers of hashtags and usertags that are particularly used for disclosing names of brands, products, or marketing campaigns in paid advertisements [37]. Additionally, we use the number of images in a post while most social media accept multiple images in a post, and the day a post was published (e.g., Sunday, Monday) since publishing time affects the popularity of advertising posts in social media [26].

- Brand features. To characterize the brand nodes, we have the business type of the brands ${ }^{3}$. Additionally, we use the number of followers, followees, and published posts to measure brand awareness [37].

\subsection{Sponsorship Estimation and Ranking}

To estimate the sponsorship score of a post, all of the GCN-encoded features, text features, and image features can be useful because many aspects of the post are considered.

For a post $i$, all of the features are concatenated as the ultimate representation $\boldsymbol{X}_{\boldsymbol{i}}$. The predicted sponsorship score $\hat{y}_{\boldsymbol{i}}$ of the post $i$ can then be generated by a linear unit with a fully-connected hidden layer as follows:

$$
\hat{y}_{i}=\mathcal{F}_{p}\left(\sigma\left(\mathcal{F}_{h}\left(X_{i}\right)\right)\right),
$$

$\overline{{ }^{3} \text { https://business.instagram.com/ }}$ 
where $\mathcal{F}_{h}(\cdot)$ and $\mathcal{F}_{p}(\cdot)$ are two fully-connected hidden layers; $\sigma(\cdot)$ is a nonlinear activation function. Therefore, the candidate posts can be ranked by the predicted sponsorship scores.

\subsection{List-wise Learning to Rank}

Since our goal is to rank posts by their likelihood scores to be sponsored posts, it is intuitive to apply learning to rank approaches to deal with the problem. More specifically, in this paper, we modify the ListMLE [36], which is list-wise learning to rank approach that can benefit overall ranking performance.

Suppose that $X$ is the set of features for posts to be ranked; $Y$ is the output space of permutations of the posts; $P_{X Y}$ is an unknown but fixed joint probability distribution of $X$ and $Y$. If a ranking function can be represented by $\hat{\boldsymbol{y}}: X \rightarrow Y$, the expected loss $R(\hat{\boldsymbol{y}})$ to be optimized can be derived as follows:

$$
R(\hat{\boldsymbol{y}})=\int_{X \times Y} L\left(\hat{\boldsymbol{y}}\left(\boldsymbol{X}_{\boldsymbol{i}}\right), \boldsymbol{y}\right) \partial P\left(\boldsymbol{X}_{\boldsymbol{i}}, \boldsymbol{y}\right),
$$

where $\boldsymbol{y} \in Y$ is a permutation; $\boldsymbol{X}_{\boldsymbol{i}} \in \boldsymbol{X} ; L\left(\hat{\boldsymbol{y}}\left(\boldsymbol{X}_{\boldsymbol{i}}\right), \boldsymbol{y}\right)$ is the 0 -1 loss between the ranked result $\hat{\boldsymbol{y}}\left(X_{i}\right)$ and the position in the permutation $y$ such that

$$
L\left(\hat{\boldsymbol{y}}\left(X_{i}\right), \boldsymbol{y}\right)= \begin{cases}1 & , \text { if } \hat{\boldsymbol{y}}\left(X_{\boldsymbol{i}}\right) \neq \boldsymbol{y} \\ 0 & , \text { if } \hat{\boldsymbol{y}}\left(X_{\boldsymbol{i}}\right)=\boldsymbol{y}\end{cases}
$$

To make the training process more efficient, candidate lists with $n$ labeled posts are sampled from the whole training space in each iteration. Given independently and identically distributed samples in a candidate list $S=\left\{\left(X_{i}, \boldsymbol{y}_{i}\right)\right\}_{i=1}^{n} \sim P_{X Y}$, we minimize the empirical loss $R_{S}$ as follows:

$$
R_{S}(\hat{\boldsymbol{y}})=\frac{1}{n} \sum_{i=1}^{n} L\left(\hat{\boldsymbol{y}}\left(\boldsymbol{X}_{\boldsymbol{i}}\right), \boldsymbol{y}_{\boldsymbol{i}}\right),
$$

where $\boldsymbol{y}_{\boldsymbol{i}}$ is the ground truth permutation.

\subsection{Temporal Regularization}

Timing is important for publishing posts in influencer marketing [26], so the redundancy between different posts with similar published times can be leveraged to improve the performance for sponsorship estimation. In addition, brands usually hire a number of influencers for a marketing campaign at a time. For example, the posts that are published at a similar time and mention the same brand name are more likely to be sponsored posts. In this paper, therefore, we conduct manifold regularization [16] by using the redundancy between the posts and their published times. Formally, the regularization loss $Q(\hat{\boldsymbol{y}})$ can be defined as:

$$
\begin{gathered}
Q(\hat{\boldsymbol{y}})=\frac{1}{n^{2}} \sum_{i=1}^{n} \sum_{j=1}^{n}\left(\left|\hat{y}_{i}-\hat{y}_{j}\right| \times \frac{w_{b}(i, j)}{\max \left(\left|l_{i}-l_{j}\right|, 1\right)}\right), \\
w_{b}(i, j)=\left\{\begin{array}{cl}
1 & , \text { if } b_{i}=b_{j} \\
10^{-1} & , \text { if } b_{i} \neq b_{j}
\end{array},\right.
\end{gathered}
$$

where $\hat{y}_{i}$ and $\hat{y}_{j}$ are the estimated sponsorship scores of the posts $p_{i}$ and $p_{j}$ mentioning the brands $b_{i}$ and $b_{j}$ at time $l_{i}$ and $l_{j} ; w_{b}(i, j)$ indicates the brand-based regularization weight. Note that the posting time difference, $\left|l_{i}-l_{j}\right|$, is measured in days. Finally, the ultimate objective for discovering sponsorship $L$ can be a combination of two loss functions as;

$$
L(\hat{\boldsymbol{y}})=R_{S}(\hat{\boldsymbol{y}})+w_{l} \cdot Q(\hat{\boldsymbol{y}}),
$$

where $w_{l}$ is the weight for manifold regularization.

\section{EXPERIMENTS}

\subsection{Experimental Dataset}

4.1.1 Dataset Construction. To evaluate our proposed model, our dataset samples influencer posts from Instagram, which is the most popular social media platform for influencer marketing [23]. Note that we implement the data collection method in [19] and comply with the Instagram policy ${ }^{4}$. To find posts that mention brand names, we first collect a set of brands on Instagram by searching branded content, i.e., sponsored posts. Note that Instagram provides the branded content tool for influencers to disclose sponsorship by showing a partnered brand name on the top of a post ${ }^{5}$. From the searched sponsored posts, we obtain 26,910 brand names. Next, we find brand mentioning posts that contain at least one brand name by searching user tags in the corresponding caption. To reduce noises in the dataset, we filter out a post if it is published by a user with less than 1,000 followers which is a generally required number of followers to be considered as an influencer ${ }^{6}$. Finally, we collect 1,601,074 brand mentioning posts that are published from 2013 to 2019 by 38,113 influencers. Note that the number of posts is exponentially grown over time; the average follower count of the influencers is 127,279 .

4.1.2 Sponsorship Labeling. Since our goal is to find sponsored posts that do not disclose paid partnerships, we first classify the posts in the dataset into two classes, including "Sponsored" and "Unknown”. We label the posts as 'Sponsored' if the posts explicitly disclose sponsored relationships by using certain keywords. More specifically, a given post is labeled as 'Sponsored' if the post either uses the branded content tool from Instagram or has one of the following hashtags, \#ad, \#sponsored, and \#paidAD, which are widely used hashtags for sponsorship disclosure in influencer marketing $[12,37]$. The remaining posts that are not identified as sponsored posts are labeled as 'Unknown'. That is, the 'Unknown' posts may contain non-sponsored and sponsored posts with no sponsorship disclosure. We label all posts in the dataset and finally have 221,710 'Sponsored' posts and 1,379,364 'Unknown' posts which account for $13.8 \%$ and $86.2 \%$, respectively. After labeling the posts, we remove all of the sponsorship-related keywords and hashtags from the posts to prevent information leakage in the experiments. Therefore, our model can detect sponsored posts without relying on such keywords. To evaluate the performance of detecting sponsored posts from the 'Unknown' posts, we further manually investigate the unknown posts. The details of manual labeling procedure are described in Section 4.4.2.

4.1.3 Heterogeneous Network. We build the heterogeneous network by using the $1,601,074$ posts which include $2,273,578$ brand

\footnotetext{
${ }^{4}$ https://help.instagram.com/325135857663734

${ }^{5}$ https://business.instagram.com/a/brandedcontentexpansion

${ }^{6}$ https://www.digitalmarketing.org/blog/how-many-followers-do-you-need-to-bean-influencer
} 
mentions. As a result, the network has 38,113 influencer nodes, 26,910 brand nodes, and 1,601,074 post nodes with 3,874,652 edges.

\subsection{Experimental Settings}

To measure the performance of the proposed SPoD, we treat the task as a one-class ranking problem and assign a relevance score for each post so that posts with higher scores are more likely to be sponsored posts. That is, the posts labeled as 'Sponsored' have relevance 1 while the 'Unknown' posts have the relevance 0 . As SPoD ranks the candidate posts by their sponsorship scores, the relevances are used to evaluate the rank quality. More specifically, we use mean average precision (MAP), mean reciprocal rank (MRR), and average precision (AP) as our evaluation metrics.

We use TensorFlow [1] to implement our model. We split the dataset into three partitions for training, validation, and testing with a ratio of 7:1:2 by randomly selecting the posts. Therefore, the ratios of sponsored posts and unknown posts on three partitions are the same. Additionally, we ensure that the same influencers are not included across the training, validation, and testing sets to avoid information leakage from learning relationships between influencers and brands (e.g., a certain influencer repetitively advertises a certain brand). We tune the parameters with the validation set and set a single hidden layer with 128 hidden nodes. The learning rate and the dropout probability are set as $10^{-3}$ and 0.5 , respectively. We set the regularization weight, $w_{l}$, as $10^{-4}$.

\subsection{Comparative Baseline Methods}

We compare the performance of the proposed model with the baseline methods in three different categories, including Ranking, Graph, and Text.

Ranking Baselines. As our model applies the learning-to-rank approach, we apply the identical feature sets of the proposed model for the ranking baselines, therefore, we can evaluate the model capability for the ranking task of the proposed model. We deploy three ranking baseline methods as follows: ListNet (LN) [6] is a list-wise learning-to-rank algorithm that exploits gradient descent on neural networks to optimize a list wise loss function. MART [14] is a pair-wise learning-to-rank algorithm that uses gradient boosted decision trees for prediction tasks. LambdaMART (LM) [5] directly optimize rank cost functions by using gradient boosted regression trees based on MART.

Graph Embedding Baselines. The baseline methods in this category only exploit the graphical structure without other information. We deploy the LINE [33] and the GCN [20] as two graph baseline methods. The baselines use the heterogeneous network as input features and disregard the text and image features.

Text Modeling Baselines. In addition to the ranking and the graph baselines, we also have two text baseline methods since influencers usually reveal paid partnerships using text. As the baselines, we deploy two state-of-the-art language models, Embeddings from Language Models (ELMo) [28] and Bidirectional Encoder Representations from Transformers (BERT) [10].

\subsection{Experimental Results}

In this section, we evaluate the performance of our proposed SPoD compared to the baseline methods with the following two steps: (i)
Table 2: Performance comparison with the baseline methods. SPoD significantly outperforms all types of baseline methods. The temporal regularization and aspect-attentive components improve the ranking performance.

\begin{tabular}{|c||c|c|c|c|c|c|}
\hline \multirow{2}{*}{ Method } & \multirow{2}{*}{ MAP } & \multirow{2}{*}{ MRR } & \multicolumn{5}{|c|}{ AP@k } \\
\cline { 4 - 7 } & & & 10 & 100 & 1000 & 10000 \\
\hline \hline LN [6] & 0.250 & 0.500 & 0.714 & 0.643 & 0.487 & 0.380 \\
LM [5] & 0.269 & 1.000 & 0.867 & 0.451 & 0.461 & 0.395 \\
MART [14] & 0.290 & 1.000 & 0.507 & 0.398 & 0.432 & 0.421 \\
\hline LINE [33] & 0.317 & 1.000 & 0.894 & 0.701 & 0.587 & 0.473 \\
GCN [20] & 0.370 & 1.000 & 0.935 & 0.744 & 0.709 & 0.566 \\
\hline ELMo [28] & 0.352 & 1.000 & 0.926 & 0.751 & 0.714 & 0.608 \\
BERT [10] & 0.376 & 1.000 & 0.947 & 0.788 & 0.755 & 0.653 \\
\hline SPoD w/o regularization & 0.558 & 1.000 & 1.000 & 0.967 & 0.956 & 0.902 \\
SPoD w/o aspect-attention & 0.573 & 1.000 & 1.000 & 0.973 & 0.960 & 0.913 \\
SPoD & $\mathbf{0 . 5 9 2}$ & $\mathbf{1 . 0 0 0}$ & $\mathbf{1 . 0 0 0}$ & $\mathbf{0 . 9 9 4}$ & $\mathbf{0 . 9 8 4}$ & $\mathbf{0 . 9 4 1}$ \\
\hline
\end{tabular}

We first examine the sponsored post ranking performance without taking into account sponsored posts in the unknown posts. (ii) We then investigate highly ranked unknown posts to evaluate the performance of detecting hidden sponsored posts in the unknown posts.

4.4.1 Sponsored Posts Ranking Performance. Table 2 shows the performance of the proposed SPoD and the baseline methods. We find that ranking baselines show poor ranking performance compared to the graph and the text baselines. Despite the graph and the text baselines only exploit the network features and the contextualized text features, respectively, these baselines outperform the ranking baselines which use all the proposed features. This is because the text features can be easily over-fitted over the complicated structures in ranking baseline methods. In other words, the ranking baselines fail to leverage the contextualized features. We also find that, in the graph and the text baselines, GCN and BERT have better rank quality than LINE and ELMo, respectively, which are adopted to our proposed post encoder.

Finally, our proposed model, SPoD, significantly outperforms all of the other baseline methods. More specifically, SPoD obtains a $57.45 \%$ improvement in mean average precision over BERT. Unlike ranking baselines, SPoD separates text and image features from GCN features and then applies attention over the features, thus effectively utilizing more important post features for ranking. Furthermore, SPoD shows a more robust ranking performance than other baselines. Note that average precision at 10,000 of SPoD is 0.941 while the performance other baseline methods tend to decrease if the ranked list size increases. Figure 4 shows precision at retrieval rates of SPoD and the other baseline methods. While the precision of the baseline methods significantly drops as the retrieval rates increase, SPoD shows highly robust performance. That is, SPoD has high ranking accuracy even while finding a large number of sponsored posts. Furthermore, we perform an ablation study by removing the temporal regularization and the aspectattention. As shown in Table 2, SPoD loses a 5.74\% and 3.21\% rank performances in the measure of MAP without using the proposed regularization and the aspect-attention, respectively. This suggests that the proposed regularization that exploits the redundancy between the posts and their published times plays an important role in discovering sponsorship of social media posts. This also reveals 


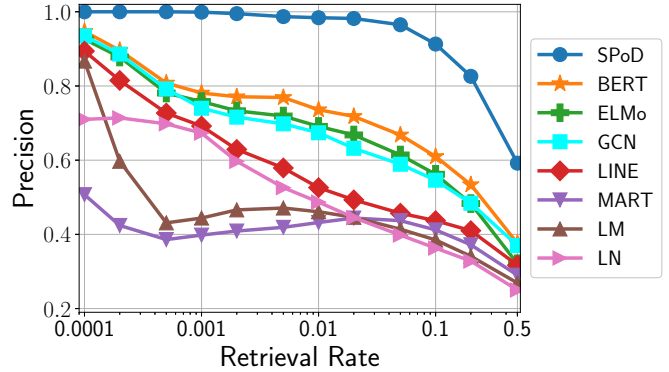

Figure 4: Precision at retrieval rates of the proposed model and the baseline methods. SPoD shows efficient and robust ranking performance compared to the baseline methods.

Table 3: Precision results on the top-ranked unknown posts. SPoD outperforms other baseline methods in detecting undisclosed sponsorship of the unknown posts.

\begin{tabular}{|c|c|c|c|c|c|}
\hline Precision@ $k$ & 10 & 50 & 100 & 150 & 200 \\
\hline GCN [20] & 0.600 & 0.540 & 0.420 & 0.380 & 0.355 \\
BERT [10] & 0.800 & 0.820 & 0.750 & 0.673 & 0.580 \\
SPoD w/o regularization & $\mathbf{1 . 0 0 0}$ & 0.960 & 0.910 & 0.873 & 0.810 \\
SPoD w/o aspect-attention & $\mathbf{1 . 0 0 0}$ & $\mathbf{1 . 0 0 0}$ & 0.950 & 0.920 & 0.860 \\
SPoD & $\mathbf{1 . 0 0 0}$ & $\mathbf{1 . 0 0 0}$ & $\mathbf{0 . 9 9 0}$ & $\mathbf{0 . 9 6 7}$ & $\mathbf{0 . 9 2 0}$ \\
\hline
\end{tabular}

that the aspect-attention effectively generates post representations by finding more important features.

4.4.2 Detecting Unlabeled Sponsored Posts. Since the goal of SPoD is to detect sponsored posts which do not clearly disclose sponsored relationship with brands, we evaluate the performance of detecting such sponsored posts by investigating the ranking results from Section 4.4.1. To this end, we first extract a set of highly ranked unknown posts and then examine their images and captions to manually label the posts. To ensure the quality of our labeling procedure, two authors of this paper have carefully read and understood the FTC's endorsement regulation, then investigated the top 200 unknown posts from each ranking result of SPoD, SPoD without regularization, BERT, and GCN. More specifically, we decide an unknown post as a sponsored post when an influencer exclusively promotes a certain product or service by expressing appreciation for sponsorship indirectly in text and holding the product to show brands in images. Cohen's kappa coefficient of our labels is 0.784 which suggests that our labeling result is highly reliable [25]. Note that there are only 9 disagreements out of the 200 posts. We consider an unknown post as a sponsored post only if both labelers agree.

Table 3 shows the precision of detecting sponsored posts from the unknown posts. The result demonstrates that SPoD is very effective in discovering the sponsored posts with the absence of sponsorship disclosure compared to the baseline methods. Note that SPoD gains $58.6 \%$ and $159.2 \%$ improvements in precision scores at 200 over BERT and GCN, respectively by achieving 0.920 precision score. This implies that the proposed post representations are remarkably useful to identify sponsorship of social media posts even if the paid partnership is not explicitly disclosed. On the other hand, using only graph structural or contextualized information may fail to detect such posts. We also find that SPoD loses $11.96 \%$ and $6.52 \%$

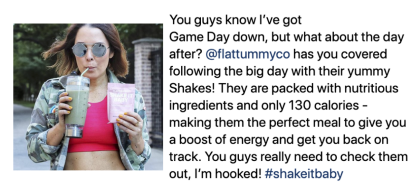

(a) Sponsored post 1

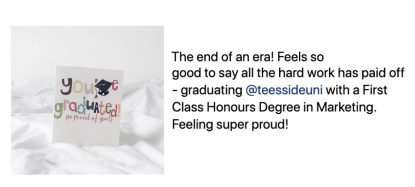

(c) Non-sponsored post 1

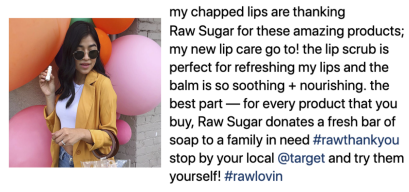

(b) Sponsored post 2

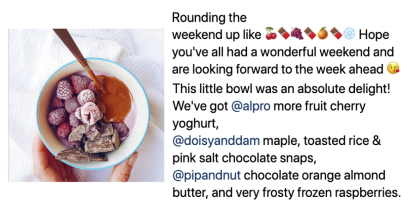

(d) Non-sponsored post 2
Figure 5: Examples of successfully detected sponsored posts with absence of sponsorship disclosure, and highly ranked non-sponsored posts.

performance in the precision at 200 without using the temporal regularization and the aspect-attention, respectively. This suggests that proposed temporal regularization significantly improves the performance of detecting undisclosed sponsorship by detecting advertising posts in the same marketing campaigns. This also reveals that the aspect-attention is useful to obtain more important knowledge from different aspects of social media posts.

Figure 5 showcases the example posts that are in the top 200 of the rank result of SPoD. The posts in Figure 5(a) and 5(b) are the sponsored posts with absence of sponsorship disclosure. In the sponsored posts, the influencers tend to describe details of the products by sharing their experience and recommending the products in the captions. In addition to the text, we observe the evidence of sponsorship in the image as they hold the products for advertising. However, the sign of sponsorship disclosure is not found from the text and hashtags. On the other hand, Figure 5(c) and 5(d) show the non-sponsored posts that are in high-rank positions. These posts may have been highly ranked due to an object in the images (e.g., products), contextual similarity, and social relation between influencers and brands.

\subsection{Analysis and Discussions}

In this section, we first study the effectiveness of the proposed model with different feature sets to understand the impact of features to discover sponsored posts. We then evaluate the performance of SPoD with the sets of posts that have different caption lengths.

4.5.1 Feature Importance. To understand the importance of each feature set in the proposed SPoD for detecting sponsored posts, we evaluate the rank performance over different feature sets. Figure 6(a) shows the performance loss of MAP scores of the models trained with the features excluding one particular node feature against the full model as the leave-one-out analysis. We find that the post features have a larger loss value than other node features since sponsored posts have distinct characteristics from non-sponsored posts [37]. Figure 6(a) also shows that the node type features have a large loss value. This suggests that social relations between influencers and brands that are indirectly learned from node types provide valuable information to detect sponsorship of posts. 


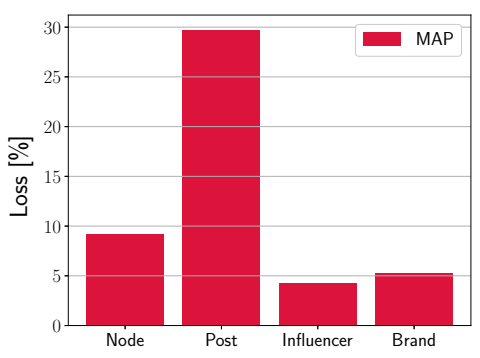

(a) Node features in GCNs

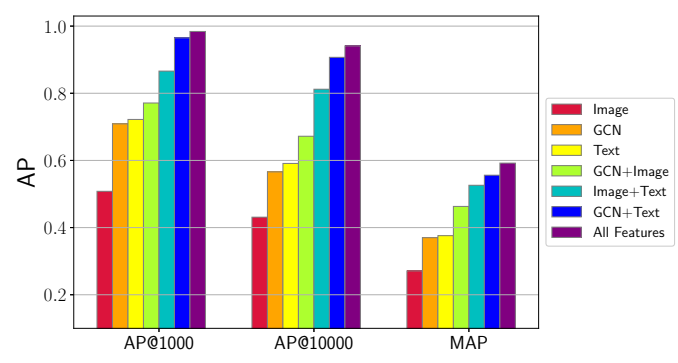

(b) Image, Text, and GCN features

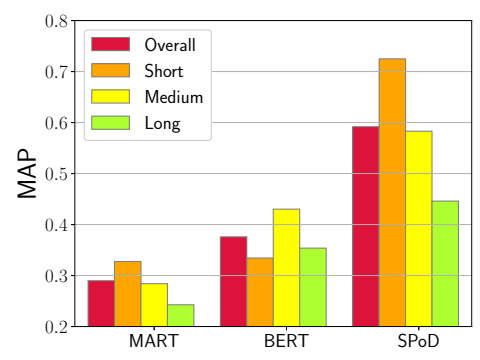

(c) Caption length of posts

Figure 6: The post node features and the text features play an important role in detecting sponsorship of posts. SPoD significantly improves the performance in the short post set.

Figure 6(b) shows the average precision scores of the proposed SPoD over (i) only image features, (ii) only GCN features, (iii) only text features, (iv) GCN and image features, (v) image and text features, (vi) GCN and text features, and (vii) All features. The result reveals that the text features significantly improve the performance while the image features contribute to the slight improvement. Note that SPoD loses $27.86 \%, 12.55 \%$, and $6.47 \%$ performance in MAP when it excludes text, GCN, and image features, respectively. This suggests that the contextualized information from captions is very useful for discovering sponsored posts. Due to the nature of paid advertisements, influencers try to recommend the products, convey detailed information of products, and to make a good impression on the brand in the text [35]. This consequently makes the paid advertisements to have distinct contextualized features from non-sponsored posts. The image features, on the other hand, have fewer benefits in discovering sponsored posts compared to the other features. Unlike the text features, which naturally show similar characteristics due to the commonality of language, images can be generated in various ways by different users, thereby making it difficult to rank the posts.

4.5.2 Caption Length of Posts. Since we find that textual information plays an important role in determining whether given social media posts are sponsored, we investigate the performance of the proposed model over different sets of posts with various caption lengths. We split the test dataset into three subsets, including short, medium, and long posts, which have less than $250(34.5 \%)$ and 500 (31.9\%), and more than $500(33.6 \%)$ characters in a caption, respectively. Note that we use the same training set for the post sets with different caption lengths thus our model can be used for posts with various caption lengths to detect sponsorship. Figure 6(c) shows the performance of SPoD and two baseline methods over the post sets in different caption lengths. By comparing the performance of MART and BERT, we observe that BERT obtains noticeable improvement in medium and long length posts. This reveals that the contextualized information greatly help detect paid partnership in the posts. However, because of the same reason, BERT fails to improve the performance on the short posts due to insufficient contextualized information from captions. As shown in Figure 6(c), the proposed SPoD outperforms the baseline methods over all the post sets in various caption lengths. Particularly, SPoD remarkably improves the performance in the short post set. This implies that SPoD effectively leverages the knowledge of both graph structures and the various features to detect the sponsorship of social media posts. For example, a sponsored post with a very short caption can be detected by our model by understanding the social relations and characteristics of adjacent influencers and brands.

\section{RELATED WORK}

Sponsorship Disclosure in Influencer Marketing. As influencer marketing has become a popular advertising method in recent years $[4,19,21]$, several previous studies show the effect of disclosing sponsorship. Evans et al. [12] find that sponsorship disclosure helps audiences recognize paid partnerships but lowers purchase intention. Stubb and Colliander [31] find that impartiality disclosure, e.g., adding "This is not sponsored post", helps generate high influencer credibility. Moreover, Evans et al. [11] investigate the effects of sponsorship text disclosure and sponsor pre-roll video advertising on YouTube. They find that the sponsor pre-roll advertising help audiences to understand sponsorship transparency. Yang et al. [37] reveal that distinct characteristics of sponsored posts, e.g., less number of usertags, longer caption than non-sponsored posts, that help exclusive promotion in advertising posts. Wojdynski et al. [35] present a metric to measure sponsorship transparency based on consumers' perceptions. However, this study only uses a very small number of sample posts and automated method has not been proposed.

Multimodal Deep Learning. Multimodal deep learning [27] is the method that can effectively generate the joint representations of different modalities by leveraging multiple sources of inputs. Since different modalities usually carry different information, combining various types of information can improve performance in learning tasks. The effectiveness of multimodal learning has led other researchers to exploit multi modalities, such as speaker identification [29], social media user profiling [19], visual question answering [22], sentiment prediction [7], depression detection [30], and speech recognition [15]. Despite the excellent performance of multimodal learning and the popularity of influencer marketing, no previous studies have yet adopted multimodal learning to discover sponsored posts.

\section{CONCLUSIONS}

In this paper, we propose a learning-to-rank model for discovering undisclosed sponsorship on social media. Our proposed model, SPoD, employs the aspect-attentive heterogeneous post encoder that incorporates graph, text, and image features to estimate their importance for representing the characteristics of social media 
posts, and then optimizes the ranking performance by using the regularization based on posting time and mentioned brands. As our proposed model effectively discover undisclosed sponsorship on social media, we strongly believe that SPoD can be deployed as follows: Companies and marketers can utilize SPoD to avoid influencers who frequently hide sponsorship in their advertising social media posts so they can collaborate with highly transparent influencers. SPoD also can be used by social media platforms to alert social media users to be conscious of undisclosed sponsorship, thereby helping them recognize the regulations on advertising posts. As a consequence, SPoD can help resolve the undisclosed sponsorship issue in influencer marketing by making influencers and brands comply with the regulations.

As future work, we intend to conduct the following studies: First, we plan to apply the proposed model to other datasets from various social media platforms as companies have started collaborating with influencers in video-based social media, including YouTube, and TikTok. In addition, we will further collect data from Instagram to include regular posts since the current dataset only contains brand mentioning posts. Next, our proposed SPoD is designed to be a ranking model to discover a set of untransparent influencers and their advertising posts with high confidence. We aim at improving the proposed model by changing it from a ranking model to a binary classifier so that the model can decide the sponsorship of a given post rather than making a ranked list of posts.

\section{ACKNOWLEDGEMENT}

We would like to thank the anonymous reviewers for their helpful comments. The work was partially supported by NSF DGE-1829071.

\section{REFERENCES}

[1] Martín Abadi, Paul Barham, Jianmin Chen, Zhifeng Chen, Andy Davis, Jeffrey Dean, Matthieu Devin, Sanjay Ghemawat, Geoffrey Irving, Michael Isard, et al. 2016. Tensorflow: A system for large-scale machine learning. In 12th USENIX Symposium on Operating Systems Design and Implementation (OSDI 16).

[2] Activate. 2018. Exploring the Brand and Influencer Relationship in Influencer Marketing. State of Influencer Marketing Study (2018).

[3] Advertising Standards Authority. 2019. The Labelling of Influencer Advertising. https:/www.asa.org.uk/uploads/assets/uploaded/e3158f76-ccf2-4e6e8f51a710b3237c43.pdf

[4] Eytan Bakshy, Jake M Hofman, Winter A Mason, and Duncan J Watts. 2011 Everyone's an influencer: quantifying influence on twitter. In Proceedings of the fourth ACM international conference on Web search and data mining. 65-74.

[5] Christopher J Burges, Robert Ragno, and Quoc V Le. 2007. Learning to rank with nonsmooth cost functions. In Advances in neural information processing systems (NIPS). 193-200

[6] Zhe Cao, Tao Qin, Tie-Yan Liu, Ming-Feng Tsai, and Hang Li. 2007. Learning to rank: from pairwise approach to listwise approach. In Proceedings of the 24th International Conference on Machine Learning (ICML). ACM, 129-136.

[7] Fuhai Chen, Rongrong Ji, Jinsong Su, Donglin Cao, and Yue Gao. 2017. Predicting microblog sentiments via weakly supervised multimodal deep learning. IEEE Transactions on Multimedia 20, 4 (2017), 997-1007.

[8] Chang-Hoan Cho. 2004. Why do people avoid advertising on the internet? fournal of advertising 33, 4 (2004), 89-97.

[9] Federal Trade Commission. 2017. The FTC's endorsement guides: What people are asking. https://www.ftc.gov/tips-advice/business-center/guidance/ftcsendorsement-guides-what-people-are-asking.

[10] Jacob Devlin, Ming-Wei Chang, Kenton Lee, and Kristina Toutanova. 2018. BERT: Pre-training of deep bidirectional transformers for language understanding. arXiv preprint arXiv:1810.04805 (2018).

[11] Nathaniel J Evans, Mariea Grubbs Hoy, and Courtney Carpenter Childers. 2018 Parenting "YouTube Natives": The Impact of Pre-Roll Advertising and Text Disclosures on Parental Responses to Sponsored Child Influencer Videos. Fournal of Advertising 47, 4 (2018), 326-346.

[12] Nathaniel J Evans, Joe Phua, Jay Lim, and Hyoyeun Jun. 2017. Disclosing Instagram influencer advertising: The effects of disclosure language on advertising recognition, attitudes, and behavioral intent. Fournal of Interactive Advertising 17,2 (2017), 138-149.

[13] The Organisation for Economic Co-operation and Development. 2019. Good Practice Guide on Online Advertising. http://www.oecd.org/officialdocuments/ publicdisplaydocumentpdf/?cote=DSTI/CP(2018)16/FINAL\&docLanguage=En .

[14] Jerome H Friedman. 2001. Greedy function approximation: a gradient boosting machine. Annals of statistics (2001), 1189-1232.

[15] Di Hu, Xuelong Li, et al. 2016. Temporal multimodal learning in audiovisual speech recognition. In Proceedings of the IEEE Conference on Computer Vision and Pattern Recognition. 3574-3582.

[16] Jyun-Yu Jiang, Pu-Jen Cheng, and Wei Wang. 2017. Open source repository recommendation in social coding. In Proceedings of the 40th International ACM SIGIR Conference on Research and Development in Information Retrieval.

[17] Louise Kelly, Gayle Kerr, and Judy Drennan. 2010. Avoidance of advertising in social networking sites: The teenage perspective. fournal of interactive advertising 10, 2 (2010), 16-27.

[18] Seungbae Kim, Jinyoung Han, Seunghyun Yoo, and Mario Gerla. 2017. How Are Social Influencers Connected in Instagram?. In International Conference on Social Informatics (SocInfo). Springer.

[19] Seungbae Kim, Jyun-Yu Jiang, Masaki Nakada, Jinyoung Han, and Wei Wang. 2020. Multimodal Post Attentive Profiling for Influencer Marketing. In Proceedings of The Web Conference 2020. 2878-2884.

[20] Thomas N Kipf and Max Welling. 2016. Semi-supervised classification with graph convolutional networks. arXiv preprint arXiv:1609.02907 (2016).

[21] Chen Lou and Shupei Yuan. 2019. Influencer marketing: how message value and credibility affect consumer trust of branded content on social media. fournal of Interactive Advertising 19, 1 (2019), 58-73.

[22] Pan Lu, Lei Ji, Wei Zhang, Nan Duan, Ming Zhou, and Jianyong Wang. 2018. R-VQA: learning visual relation facts with semantic attention for visual question answering. In Proceedings of the 24th ACM SIGKDD International Conference on Knowledge Discovery \& Data Mining. ACM, 1880-1889.

[23] MarketingHub. 2019. The State of Influencer Marketing 2019 : Benchmark Report. Benchmark Report (2019).

[24] Masoud Mazloom, Robert Rietveld, Stevan Rudinac, Marcel Worring, and Willemijn Van Dolen. 2016. Multimodal popularity prediction of brand-related social media posts. In Proceedings of the 24th ACM international conference on Multimedia (MM). ACM, 197-201.

[25] Mary L McHugh. 2012. Interrater reliability: the kappa statistic. Biochemia medica: Biochemia medica 22, 3 (2012), 276-282.

[26] Sérgio Moro, Paulo Rita, and Bernardo Vala. 2016. Predicting social media performance metrics and evaluation of the impact on brand building: A data mining approach. Journal of Business Research 69, 9 (2016).

[27] Jiquan Ngiam, Aditya Khosla, Mingyu Kim, Juhan Nam, Honglak Lee, and Andrew Y Ng. 2011. Multimodal deep learning. In Proceedings of the 28th international conference on machine learning (ICML-11). 689-696.

[28] Matthew E. Peters, Mark Neumann, Mohit Iyyer, Matt Gardner, Christopher Clark, Kenton Lee, and Luke Zettlemoyer. 2018. Deep contextualized word representations. In Proc. of NAACL.

[29] Jimmy Ren, Yongtao Hu, Yu-Wing Tai, Chuan Wang, Li Xu, Wenxiu Sun, and Qiong Yan. 2016. Look, listen and learn-a multimodal LSTM for speaker identification. In Thirtieth AAAI Conference on Artificial Intelligence.

[30] Guangyao Shen, Jia Jia, Liqiang Nie, Fuli Feng, Cunjun Zhang, Tianrui Hu, TatSeng Chua, and Wenwu Zhu. 2017. Depression Detection via Harvesting Social Media: A Multimodal Dictionary Learning Solution.. In IfCAI. 3838-3844.

[31] Carolina Stubb and Jonas Colliander. 2019. "This is not sponsored content"-The effects of impartiality disclosure and e-commerce landing pages on consumer responses to social media influencer posts. Computers in Human Behavior (2019).

[32] Christian Szegedy, Vincent Vanhoucke, Sergey Ioffe, Jon Shlens, and Zbigniew Wojna. 2016. Rethinking the inception architecture for computer vision. In Proceedings of the IEEE conference on computer vision and pattern recognition (CVPR). 2818-2826.

[33] Jian Tang, Meng Qu, Mingzhe Wang, Ming Zhang, Jun Yan, and Qiaozhu Mei. 2015. Line: Large-scale information network embedding. In Proceedings of the 24 th international conference on world wide web. International World Wide Web Conferences Steering Committee, 1067-1077.

[34] Ashish Vaswani, Noam Shazeer, Niki Parmar, Jakob Uszkoreit, Llion Jones, Aidan N Gomez, Łukasz Kaiser, and Illia Polosukhin. 2017. Attention is all you need. In Advances in neural information processing systems. 5998-6008.

[35] Bartosz W Wojdynski, Nathaniel J Evans, and Mariea Grubbs Hoy. 2018. Measuring sponsorship transparency in the age of native advertising. Fournal of Consumer Affairs 52, 1 (2018), 115-137.

[36] Fen Xia, Tie-Yan Liu, Jue Wang, Wensheng Zhang, and Hang Li. 2008. Listwise approach to learning to rank: theory and algorithm. In Proceedings of the 25th International Conference on Machine Learning (ICML). ACM.

[37] Xiao Yang, Seungbae Kim, and Yizhou Sun. 2019. How do influencers mention brands in social media? sponsorship prediction of Instagram posts. In Proceedings of the 2019 IEEE/ACM International Conference on Advances in Social Networks Analysis and Mining. 101-104. 